\title{
A Survey on Semantic Web based E-learning
}

\author{
Pradeep Kumar Tiwari \\ Department of CSE \\ MANIT Bhopal (India)
}

\author{
Jaytrilok Choudhary \\ Department of CSE \\ MANIT Bhopal (India)
}

\author{
Deepak Singh Tomar \\ Department of CSE \\ MANIT Bhopal (India)
}

\begin{abstract}
E-learning is method of distance learning using electronic media, in which learner and instructor interacts via learning content present on web. Semantic web is extension of current web. Semantic web attains importance now a day. For implementing the effective e-learning system, Semantic Web appears as a promising technology. Now, it becomes a need to use semantic nature of web in e-learning. Semantic web based e-learning is next generation of e-learning. This paper presents the different kind of e-learning models.
\end{abstract}

\section{Keywords}

E-learning, Semantic web, recommendation system, personalization, semantic portal, ontology.

\section{INTRODUCTION}

E-learning is delivered just in time education, which provides interactive, non-linear, systematic, distributed, personalized and dynamic learning content to user in real time. By making Use of e-learning system learner interact with expert [1].

E-learning is a distance learning process, in which student can access teaching material electronically according to his interest or need and students-teachers can communicate within and with each other electronically. Learning performance of learner depend on characteristics of particular learner profile, goal, interest, previous knowledge level etc.

Reason of emergence of semantic web is that, in semantic web information can be expressed in the form which is easily understandable by machine. Semantic web can also be used for the implementing e-learning system [2]. For making the Web machine understandable, Tim Berners-Lee proposed a Semantic Web model by including semantics nature to the Web [3]. Layer architecture of semantic web is shown in figure 1 [3].

Unicode and URI (Uniform Resource Identifier) are present in bottom layer of Semantic Web layered architecture. Unicode is encoding scheme to represent the human readable language on the Web For uniquely identify the resource on the Web URI is used. Resource can be anything; it can be web accessible material or Abstract thing like a person name. URI is the superset of URL. In URL (Uniform Resource Locator), network location of the resource is present.

Above the Unicode and URI layer, there is XML layer. Web documents can be written with help of XML language. XML is used for sending documents across the Web in user define vocabulary.

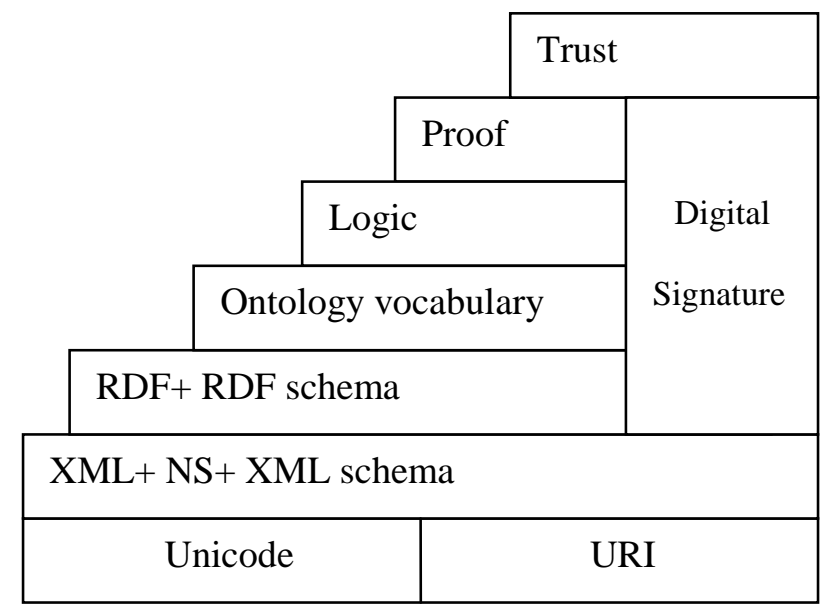

Fig 1: Semantic Web Layered Architecture

$\mathrm{RDF}$ is a basic type of data model by which one can write statements about Web objects. It is located on top of the XML layer. The RDF has an XML-based syntax. RDF Schema is based on RDF. It provides modelling primitives such as class/subclass, property/sub property relationships, domain and range restrictions etc. RDF Schema can be used as a primitive language for writing ontology's. For defining the complex relationship among the concepts, ontology layer is used.

Actual deductive process, as well as the representation of proofs in Web languages and proof validation involves in proof layer. At last trust will be obtained through the use of Digital signatures, certification agencies and consumer bodies [3].

\section{FEATURES SUPPORTED BY AN E- LEARNING SYSTEM}

According to Dawn G. Gregg following features should be provided by the learning system to user [4].

1) Active learning: The content of the learning course must maintain quality of interactively, responsiveness so that learner can use the system with efficiently.

2) Personalized: Flexibility and adaptability of the learning material should be up to such extent so that it can be change according to individual's user profile. The user profile is consisting of level of understanding, prior knowledge and learning style of the user. 


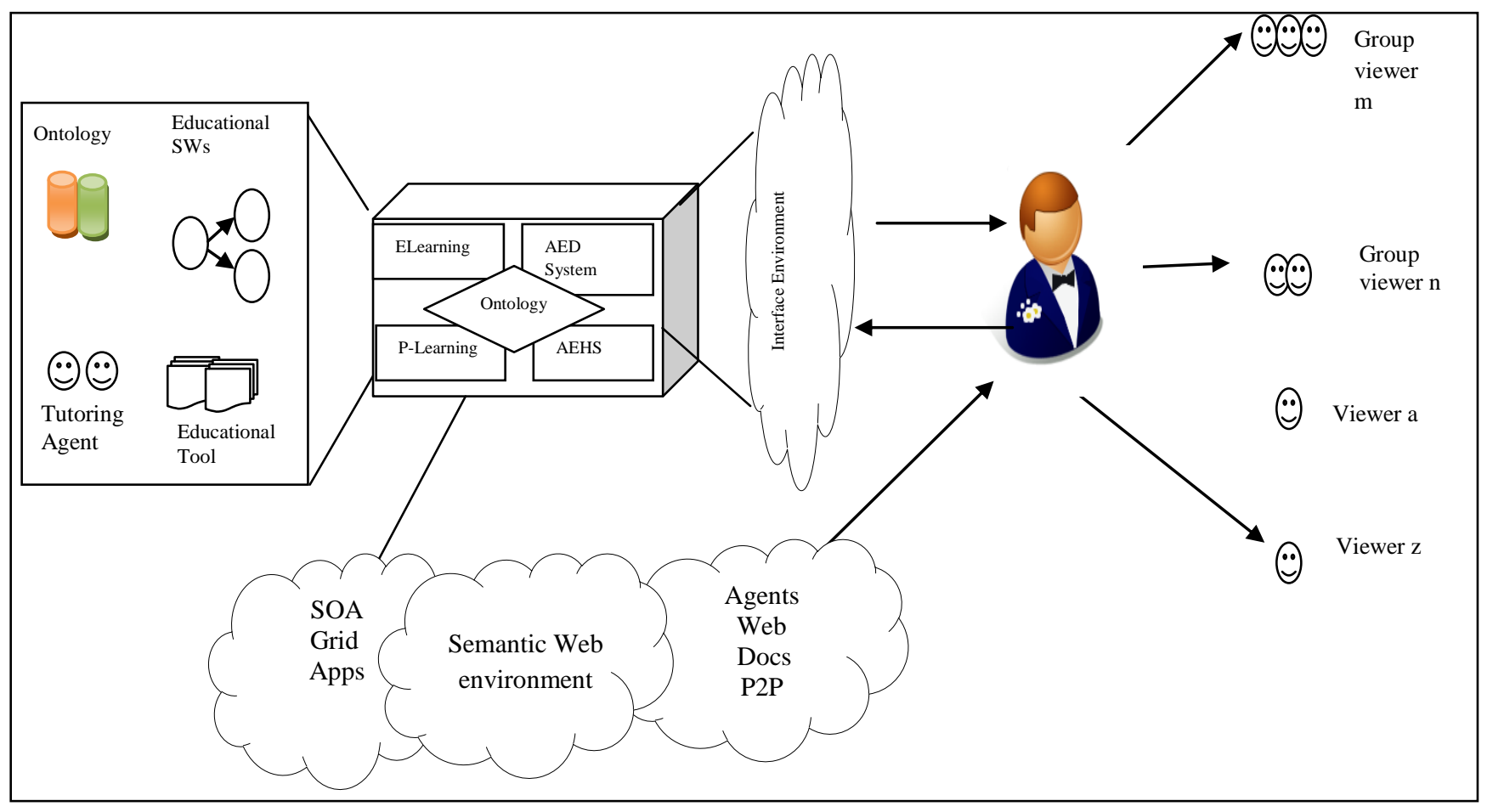

Fig 2: Reference model of a semantic web-based educational system

3) Delivery plan: It deals with how to make content efficient and then present to user so it should help the instructor in information collection then in planning how content should be presented to the user so that user can obtain maximum benefit from courses.

4) Resource Location: Location of resource on the web should be such that, it can be updated easily and efficiently whenever needed.

\section{REFERENCE MODEL FOR E- LEARNING SYSTEM}

Fig. 2 describes a reference model of a semantic web based educational system [5]. Each component concerning semantic web-based educational systems is discussed as follows:

Role: In semantic web-based educational systems, there are several educational activities these activities are distributed according to the roles.

Interface environment: It provides interface for interaction between the SWBES and the users.

Educational resources: these are learning objects related to a specific educational system, such as topic, examples, problems etc.

SWBES: It represents the next generation of web-based educational systems that aim at providing several improvements on the quality of services by the use of semantic web technology.

Semantic web environment: It represents the interaction environment available to SWBES and users to discover, browse, select and invoke resources on the web according to several technologies and architectures semantically described

\section{LITERATURE REVIEW}

Chih Ming Chen et. al. [6] has proposed an ontology-based concept maps for planning an appropriate-learning path for individual learner. For generating the course map of the subject, they had used the concept of fuzzy clustering [7]. They also calculate the correlation between the course concepts. After finding the fuzzy clusters and correlation between them, concept map can be generated with the help of proposed formula.

A personalize information retrieval approach in an e-learning environment has proposed by Zhuhadar et al. [8]. In the proposed architecture, domain ontology is encoded into tree structure with the help of OWL (web ontology language). This tree structure is called semantic domain. After that semantic learner profile is extracted from the semantic domain according to the visited nodes (concepts) by the user with the help of bottom up pruning. Time interval during which, user visit node is a controlling parameter for memory span of the user profile. After that documents are clusters into groups by the one of various hierarchical clustering algorithms. Similarity between user profile and cluster find and most similar cluster is used as a recommended cluster for the user. Now when user searches a query, the default search results come. These default search results are re rank based on whether the search output documents are in user profile or recommended cluster. User profile documents have highest priority than recommended cluster documents and rest of the documents. Experimental results show that the personalized semantic search improve the precision of search.

Antony et al. [9] proposed an adaptive e-learning system. Initially user interest towards different courses is calculated with the help of SMS triggered E-Assessments and then classify the different users based on the assessment score.

With the help of the domain ontology similarity between different users is measured and classifies them in different clusters. Finally, adaptive recommendations for individual users provided based on nearest neighbourhoods in the form of resources, assignments and further E-Assessment. 
Yanyan $\mathrm{Li}$ et. al. [10] proposed a knowledge portal for supporting e-learning system which utilizes semantic nature of web. Knowledge portals focus on the construction, acquisition, transmission or the management of knowledge, for information providing and accessing. This portal is made up of following modules.

Reference ontology: It is made up of knowledge algebra and knowledge base. Knowledge algebra is concepts or word while knowledge base is the relationship or properties through which they are connected. Through these relationships different concept are connected semantically. By use of semantic web technology, semantic heterogeneous learning resources are represented in a consistent and meaningful scheme.

Knowledge refinement: It includes two subtask relevancy checking and redundancy removal. In relevancy checking, it is checked that concept is relevant or not, to the topics given in ontology database. Then the knowledge is classified in different classes using SVM methodology [11]. The knowledge engineer will help in redundancy removal process.

Knowledge retrieval: input query is annotated according to user profile and interest then by using ontology, semantically relevant information is retrieved.

This system provides qualitative interface for different type of user to exploit the learning resources. System may be further improved by use of semantic link network for organisation of semantic resources.

Shrivastava et al.[12] proposed a semantic web based elearning model based on agents. For implementing the elearning system there are many agents as described by Dawn G. Gregg in his paper [4]. These are Instruction Agent, Lesson Planning Agent, Resource Location Agent, Personalization Agent, Learner Centred Agent, and Collaboration Agent. These agents were used to describe an elearning system. Semantic web is used by some of these agents in order to make the-learning system more responsive and interactive. Shrivastava used these agents to describe the e-learning system. In proposed model there are two users instructor and learner. Both use the appropriate agent for proving and getting the-learning resource.

Huang et al.[13] proposed a method for reducing the time and complexity for retrieving the-learning material and providing personalized learning path to the learner. They proposed Educational Knowledge Service System (EKSS). In EKSS knowledge is represented as a three dimensional point with coordinate

<knowledge-category, knowledge-level, knowledge-location> [14]

In EKSS, connection between various learning resources provided through OWL. Personalized recommendation can be described in DAMLS, and are accessible via WSDL and SOAP. When learner fired query, EKSS analyses is prerequisite for relationships of concepts and for a particular concept. If all the pre requisite concepts have been covered by the user then that concept recommend to user.

The EKSS system is made up of three modules knowledge service interface, knowledge processing unit and personnel knowledge search engine. Through Knowledge service interface module, user interacts with system by using view, store, access, and search. Knowledge processing unit integrate and organise decentralized information, using ontology measuring and overlapping elimination [15]. Personnel knowledge search engine personalize knowledge according to learner profile and support to search learning path suitable for user. Knowledge service functionality has been integrated with semantic web service.

Chih Ming Chang [16] gave personalized E-learning system with self regulated learning, to improve Performance of elearning system. It is based on Self regulating model given by Zimmerman et. al.[17].

Zimmerman's Model has four learning process, which are self evaluation and analysis, goal setting and strategy planning, strategy implementation and monitoring, strategy outcome analysis.

Chih Ming Chang uses Zimmerman model as well as three more agents which are personalized interface agent, interactive teaching agent and personalized SRL agent. Personalized interface agent provides interface for learner to communicate with PELS and SRL agent. Interactive teaching interface provides interface for teacher to monitor learner status, give hint to learner, and give answer of the question asked by learner. It also provide interface to learner through which he can communicate with teacher about his problem.

System component of [16] include following

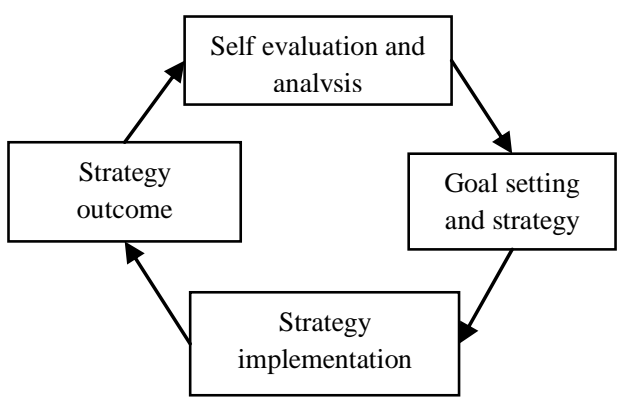

Fig 3 : Self-regulated learning proposed by Zimmerman [17]

Personalized self regulated learning agent:-it has four module namely self monitoring module, self evaluation module, self regulation learning competence indicator module and self regulated learning portfolio learning database. Self monitoring module is used for choosing best strategy for learning and work on how to improve learning. Self evaluation module used to evaluate outcome of the course which completed recently. SRL competence indicator module analyse SRL indexes which is in SRL portfolio database, and it also calculates learning performance index, ability index, achievement index. It also has database SRL portfolio database which is used to record SRL process during learning.

Interactive teaching agent: has two modules namely hint message module and which helps learner to understand course content, and Q\&A module used for answering query fired by student.

Self regulated learning interactive interface agent:-Using this module interaction between learner and system take place by use of self regulated learning competence index.

Pre-test and post-test are used to check the knowledge of the user before and after course. Pre-test determine the awareness about the course initially. Post-test determines learning ability of users, it also compare the difference of learning performance of users. 
This system helps to promote self regulated learning ability of users. it helps learner to determine learning goal and improving performance. Interactive teaching agent assists in learning. Learning performance also increased.

Hiekata, et al. [18] proposed a semantic web based e-learning framework using the ShareFast. Share Fast is an open source application for document management system. Framework is consisting of five modules which are student, teacher, shearFast system, IT infrastructure and design software.

The key concept of ShareFast is to visualize learning processes into hierarchical workflows, and manage documents by attaching them to the related task in workflow, together with many supporting tools provided. ShareFast is a semantic web based application. Teacher can create a workflow for learning using Share Fast server application and then student can follow the workflow for learning. Client Share Fast records the log history of the student and sends to the server ShareFast so that teacher can set the workflow for student according to log history. Teacher and student can interact using discussion thread.log history include input given by student during learning. By making analysis of log file of student/class, teacher can understand learning behaviour of student/class, and after analysing log file, teacher can modify learning content according to student/class quality. Discussion thread plays significant role when tutor and student are away from each other.

Hiekata conducted a study which concludes that by analysing result of one group and modify content according to analysis result, and then present it to second group. it will improve the learning quality of second group.

Kalla Madhu Sudhana et. Al. [19] proposed an ontological approach to support personalized e-learning system, which presents learning material according to the learner's preference. This model is made up of two part learning repository mo and learner models, both of these parts are represented through ontologies.

This System is consisting of following modules.

Domain management module: This module acts as interface between learner repository and personalized learning module. This module is used for updating and maintaining extension in repositories and provides a way to user to access material sequentially.

Learner management module: This module manages registration and record activity of e-learner and monitors learning process. At last it generates report of e-learner.

Personalized learning support module: This module evaluate whether the document is relevant to the input query and user preferences.

Domain ontology module: It is use to manage learning material to user side. By use of domain ontology module it is decided that how much a material is important to user. This ontology is used for annotation, structuring and organisation of learning material.

User model ontology module: This module contains information provided by learner via user interface, learner profile and external environment.

Rank learning material based on similarity between query and related subject. They Used precision and recall parameter to calculate performance.

\section{RESEARCH GAPS}

Semantic e-learning is a group of technologies, protocols, processes and techniques. So, these types of systems are complicate to implement in the current scenario. However, it is sure that the next generation of e-learning is Semantic Web based-learning. Thus, issues and challenges must be handling before Semantic e-learning technology gain popularity.

Interoperability is the ability to exchange data and enables information and knowledge sharing. It appears as the mean for accomplishing the inter-linking of information, systems, applications and ways of working. So, interoperability features as a very important requirement for future e-learning.

Construction of e-learning ontology's is not a simple task. So, involvement of expert is needed for ontology construction because experts have very much knowledge and focused thinking about the topic whose ontology is to be constructed. Sometimes ontological decisions contained in the data repositories may not similar to those of the user wants. This happens in many e-learning environments, where the differences of the various notions may often be indistinguishable. This problem can be solved by using multiple ontology, separately for each user profile in place of a single universal ontology.

Information sharing should be such that it should follow personal data protection principles, laws and regulations. Digital data's collection, storage, processing, transfer, and sharing are used for this.

\section{CONCLUSION}

Main problem in e-learning technology is lack of accuracy, time limitation, information overload and cost. There should be mechanism to structure the huge amount of e-learning resources and make it readily accessible and reusable in a personalized way. This access should be assisted for the various types and levels of the e-learning community, whether they belong to professional training, academic programs, lifelong learners or others.

\section{REFERENCES}

[1] Drucker, P. 2000 "Need to Know: Integrating e-learning with High Velocity Value Chains," A Delphi Group White Paper.

[2] Stojanovic, L., Staab, S., Studer, R. 2001 "e-learning based on Semantic Web" World Conference on the WWW and Internet.

[3] Berners-Lee, T., Hendler, J. and Lasilla, O. 2001 The Semantic Web, Scientific American May.

[4] Gregg, D.G. 2007 "e-learning agents", Learning Organization, Vol. 14 Issue: 4, pp.300 - 312.

[5] Dunkel, J., Bruns, R., Ossowski, S. 2006 Semantic elearning Agents, Supporting e-learning by Semantic Web and Agents Technologies, Enterprise Information Systems, 237244.

[6] Chen, C.M. and Peng, C.J. 2007 "Personalized e-learning System based on Ontology-based Concept Map Generation Scheme," Seventh IEEE International Conference on Advanced Learning Technologies (ICALT).

[7] Bezdek, J. C. 1981 Pattern Recognition with Fuzzy Objective Function Algorithms, Plenum. 
[8] Zhuhadar, L., and Nasraoui, O. 2008 Personalized cluster based semantically enriched web search for e-learning. Proceedings of the 2nd international workshop on Ontology's and information systems for the semantic web ACM.

[9] Antony, J., Thottupuram, F.R., Thomas S., John, M.V. 2012 "Semantic web based adaptive e-learning triggered through short message services." Computer Science \& Education (ICCSE), 2012 7th International Conference on IEEE.

[10] Li, Y., and Dong, M. 2008 "Towards a Knowledge Portal for e-learning based on semantic Web." Advanced Learning Technologies, 2008.ICALT'08.Eighth IEEE International Conference on IEEE.

[11] Vapnic, V., Golowhich, S., \& Smola, A. 1996 "Support vector method for function Approximation, registration and signal processing system, Cambridge MA: MIT press 281-287.

[12] Shrivastava, G., Sharma, K. and Bawankan, A. 2012 "A new framework semantic web technology based elearning." Environment and Electrical Engineering (EEEIC), 11th International Conference on IEEE.

[13] Huang, T., Yang, Z., Liu, Q., Li, X., Wu, B., Liu, S. \& Zhao, G. 2006, "Semantic Web-based Educational Knowledge Service System (EKSS) for e-learning". First International Conference on Communications and
Networking in China China Com'06 ; Beijing, China (pp. $1-5)$.

[14] Burstein, M., Bussler, C. 2005 A Semantic Web Services Architecture, IEEE Internet comupting,september,

[15] Smola, A.J., \& Schölkopf, B. 2004. A tutorial on support vector regression. Statistics and computing, 14(3), 199222.

[16] Chen, C.M., 2009. Personalized e-learning system with self-regulated learning assisted mechanism for promoting learning performance, Expert system with applications $36,8816-8829$.

[17] Zimmerman, B. J., Bonner, S., and Kovach, R. 1996. Developing self-regulated learner: Beyond achievement to self-efficacy. Washington: APA.

[18] Hiekata, K., Yamato, H., Rojanakamolsan, P., Oishi, W. 2007. "Design engineering educational framework using sharefast: a semantic web-based e-learning system." Information Technology, ITNG'07.Fourth International Conference on. IEEE.

[19] Sudhana, K. M., Raj, V. C., and Sikamani, K. T. 2013. An Ontological Approach to Support Personalized E-Learning System. 